\title{
QUATÉRNIONS E AS ROTAÇÕES NO ESPAÇO
}

\section{QUATERNION AND THE ROTATION IN SPACE}

Amanda Santos Silva, Juliano Ferreira de Lima, Antônio Carlos Tamarozzi.

Universidade Federal de Mato Grosso do Sul - UFMS, Curso de Matemática Licenciatura, Três Lagoas, MS.

E-mail: assilva010@gmail.com

RESUMO - Os Quatérnions foram criados em 1843 por W. R. Hamilton e sua utilização, embora não seja muito divulgada, não é recente. Basicamente os Quatérnions podem ser vistos como uma extensão da álgebra dos números complexos, na qual se tem três componentes imaginários ao invés de um, podendo ser representado por $\dot{a}=a+$ $a_{x} \vec{\imath}+a_{y} \vec{\jmath}+a_{z} \vec{k}=(a, \vec{a})$, onde $a$ é um escalar e $\left(a_{x}, a_{y}, a_{z}\right)$ são os componentes do vetor $\vec{a}$. Uma vez especificadas algumas propriedades e operações elementares, a partir dessa definição, pode-se provar que esse conceito inicial, permite estabelecer a relação da álgebra dos Quatérnions. Nesse contexto, o objetivo deste trabalho é apresentar alguns conceitos básicos relativos à Álgebra dos Quatérnios, apontando alguns aspectos dessa representação.

Palavras-chave: Quatérnions; Anéis não Comutativos; Números Complexos; W. R. Hamilton.

\section{ABSTRACT - ABSTRACT}

The Quaternions were created in 1843 by W. R. Hamilton and its use, although not much publicized, it is not new. Basically, quaternions can be seen as an algebraic extension of complex numbers, in which it has three imaginary components instead of one, may be represented by $\dot{a}=a+a_{x} \vec{\imath}+a_{y} \vec{\jmath}+a_{z} \vec{k}=(a, \vec{a})$, where $a$ is a scalar and $\left(a_{x}, a_{y}, a_{z}\right)$ are the vector components $\vec{a}$.

Once specified some properties and basic operations, from this definition, we can prove that this initial concept, allowing the correlation between the algebra of quaternions. In this context, the aim of this paper is to present some basic concepts related to the Algebra of Quaternions, pointing out some aspects of this representation.

Keywords: Quaternions; Noncommutative Rings; Complex Numbers; W. R. Hamilton. 


\section{INTRODUÇÃO}

Segundo Boyer (1974), William Rowan Hamilton apresentou o primeiro conceito moderno dos complexos como pares ordenados de reais e tentou generalizar esta ideia para o espaço tridimensional. Após inúmeras tentativas verificou-se que não era possível a existência de um complexo

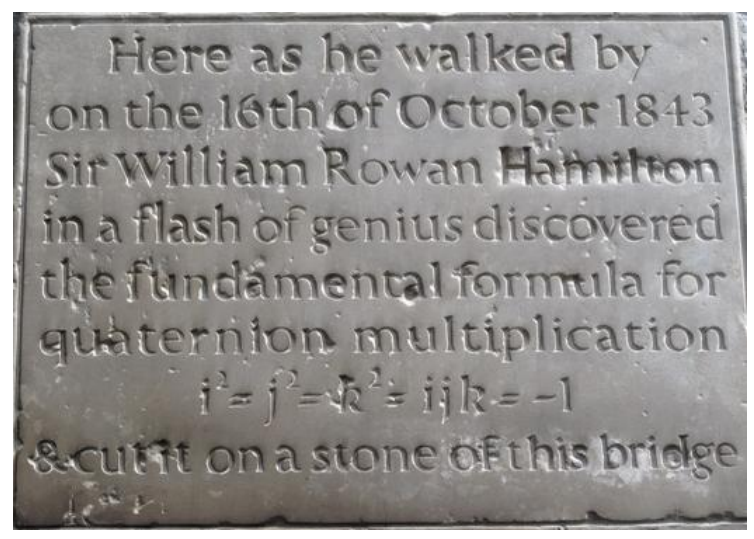

Figura 1. Dizeres entalhados por Hamilton Fonte: Ingenious Ireland (2011).

A álgebra dos quatérnions foi a primeira álgebra não-comutativa da história.

O objetivo deste estudo realizado pelo grupo Pet foi aprofundar o conhecimento sobre os conceitos de Álgebra, sobre todas as propriedades dos anéis não comutativos e estender o conhecimento do Conjunto dos Complexos.

\section{METODOLOGIA}

Os Quatérnions são definidos no espaço $\mathbb{R}^{4}$, sendo algumas vezes simbolizados por $\mathbb{H}$ em homenagem ao seu tridimensional. Com isso, Hamilton descobriu os quatérnios, que é uma Álgebra de dimensão quatro sobre o corpo dos números reais e que possui todas as propriedades de um corpo, exceto a comutatividade da multiplicação. Dizem que Lorde Hamilton teve a ideia de definir o produto de Quatérnions num passeio que fez com a Lady Hamilton.

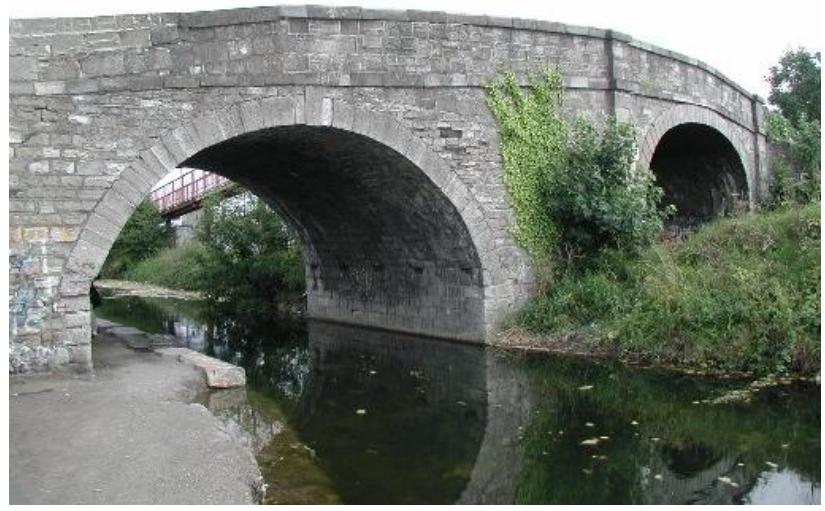

Figura 2."Quatérnion Bridge"

Fonte: Brougham Bridge (2004).

criador. Os quatérnios podem ser interpretados de várias maneiras, dentre elas: como um vetor de dimensão quatro ou um número complexo com três unidades imaginárias, Considerando o escalar 1 e os versores $\vec{\imath}, \vec{\jmath}, \vec{k}$ como base do espaço de quatérnios pode-se representar um quatérnio genérico, de forma que $\dot{a} \in \mathbb{H}$, por: 


$$
\dot{a}=a+a_{x} \vec{\imath}+a_{y} \vec{\jmath}+a_{z} \vec{k}=a+\vec{a}=(a, \vec{a})=\left(a, a_{x}, a_{y}, a_{z}\right),
$$

onde $a, a_{x}, a_{y}$ e $a_{z}$ são escalares reais e $a_{x}, a_{y}$ e $a_{z}$ são componentes do vetor $\vec{a}$.

Podemos notar que na equação 1 temos quatro modos para representar os quatérnios. Uma característica dos quatérnios é que ele pode ser usado tanto para representar um escalar, um número complexo na forma $z=a+b i$ quanto um vetor do $\mathbb{R}^{3}$. Fazendo $q=0$ teremos um vetor, chamado também de quatérnio puro; considerando $a_{y}=a_{z}=0$ teremos um número complexo e fazendo $a_{x}=a_{y}=a_{z}=$ 0 teremos um escalar.

Primeiramente definiremos algumas propriedades de vetores. Sejam $\vec{u}=$ $(x, y, z)$ e $\vec{v}=\left(x_{1}, y_{1}, z_{1}\right)$ dois vetores no $\mathbb{R}^{3}$, Assim, podemos definir:

- Produto escalar
O produto Escalar de $\vec{u}$ por $\vec{v}$, representado por $u \cdot v$, é o escalar definido por

$$
\begin{aligned}
\vec{u} \cdot \vec{v} & =(x, y, z) \cdot\left(x_{1}, y_{1}, z_{1}\right) \\
& =x x_{1}+y y_{1}+z z_{1}
\end{aligned}
$$

Temos da definição que o produto escalar é comutativo: $\vec{u} \cdot \vec{v}=\vec{v} \cdot \vec{u}$. Esse produto é também conhecido como produto interno.

- Produto vetorial

\section{O produto}

vetorial de $\vec{u}$ por $\vec{v}$, representado por $\vec{u} \times$ $\vec{v}$, é o vetor definido por

$$
\begin{gathered}
\vec{u} \times \vec{v}=\left|\begin{array}{ccc}
\vec{\imath} & \vec{\jmath} & \vec{k} \\
x & y & z \\
x_{1} & y_{1} & z_{1}
\end{array}\right| \\
=y z_{1} \vec{\imath}+z x_{1} \vec{\jmath}+x y \vec{k}-x_{1} y \vec{k}-y_{1} z \vec{\imath}-z_{1} x \vec{\jmath} \\
=\left(y z_{1}-y_{1} z\right) \vec{\imath}+\left(z x_{1}-z_{1} x\right) \vec{\jmath}+\left(x y_{1}-x_{1} y\right) \vec{k}
\end{gathered}
$$

Onde $\vec{\imath}, \vec{\jmath}$ e $\vec{k}$ são versores e representam os eixos Ox, Oy e Oz, respectivamente.

Assim como nos números complexos onde $i$ é a unidade imaginária $\left(i^{2}=-1\right)$, os três componentes imaginários do quatérnio, denominados imaginários principais possuem a mesma propriedade. Além desta propriedade os produtos, dois a dois, de $i, j$ e 
$k$, seguem a mesma regra do produto vetorial. Deste modo podemos estabelecer as seguintes relações:

$$
\begin{gathered}
i^{2}=j^{2}=k^{2}=-1 . \\
i j=-j i=k . \\
k i=-i k=j .
\end{gathered}
$$$$
j k=-k j=i \text {. }
$$

Que também pode ser representado pela tabela a seguir:

\begin{tabular}{|c|c|c|c|}
\hline & $\boldsymbol{i}$ & $\boldsymbol{j}$ & $\boldsymbol{k}$ \\
\hline $\boldsymbol{i}$ & -1 & $k$ & $-j$ \\
\hline
\end{tabular}

\begin{tabular}{|c|c|c|c|}
\hline$j$ & $-k$ & -1 & $i$ \\
\hline $\boldsymbol{k}$ & $j$ & $-i$ & -1 \\
\hline
\end{tabular}

Como consequência das equações (2), segue que:

$$
\begin{array}{r}
i j k=k^{2}=-1 \\
i k j=(-j) j=1 \\
k j i=(-i) i=1
\end{array}
$$

Dado o quatérnion $\dot{a}$, podemos definir algumas propriedades fundamentais:

- Conjugado de $\dot{a}$

$$
\dot{a}^{\prime}=a-a_{x} \vec{\imath}-a_{y} \vec{\jmath}-a_{z} \vec{k}=a-\vec{a}=(a,-\vec{a})=\left(a,-a_{x},-a_{y},-a_{z}\right)
$$

- Norma de $\dot{a}$

$$
|\dot{a}|=\sqrt{a^{2}+a_{x}^{2}+a_{y}^{2}+a_{z}^{2}}
$$

- Quatérnion unitário

$$
|\dot{a}|=\sqrt{a^{2}+a_{x}^{2}+a_{y}^{2}+a_{z}^{2}}=1
$$

Sejam $\dot{a}, \dot{b} \in \mathbb{H}$, assim definimos:

- Soma de Quatérnions

$$
\begin{gathered}
\dot{a}+\dot{b}=(a, \vec{a})+(b, \vec{b}) \\
=\left(a+a_{x} \vec{\imath}+a_{y} \vec{\jmath}+a_{z} \vec{k}\right)+\left(b+b_{x} \vec{\imath}+b_{y} \vec{\jmath}+b_{z} \vec{k}\right) \\
=(a+b)+\left(a_{x}+b_{x}\right) \vec{\imath}+\left(a_{y}+b_{y}\right) \vec{\jmath}+\left(a_{z}+b_{z}\right) \vec{k} \\
=(a+b, \vec{a}+\vec{b})
\end{gathered}
$$

- Produto de quatérnions

$$
\begin{gathered}
\dot{a} \dot{b}=(a, \vec{a}) \cdot(b, \vec{b}) \\
=\left(a+a_{x} \vec{\imath}+a_{y} \vec{\jmath}+a_{z} \vec{k}\right) \cdot\left(b+b_{x} \vec{\imath}+b_{y} \vec{\jmath}+b_{z} \vec{k}\right)
\end{gathered}
$$




$$
\begin{gathered}
=a b+\left(a b_{x}\right) \vec{\imath}+\left(a b_{y}\right) \vec{\jmath}+\left(a b_{z}\right) \vec{k}+\left(a_{x} b\right) \vec{\imath}+\left(a_{x} b_{x}\right) \overrightarrow{\imath \imath}+\left(a_{x} b_{y}\right) \vec{\imath}+\left(a_{x} b_{z}\right) \vec{\imath} \vec{k} \\
+\left(a_{y} b\right) \vec{\jmath}+\left(a_{y} b_{x}\right) \vec{\jmath} \vec{\imath}+\left(a_{y} b_{y}\right) \overrightarrow{\jmath \jmath}+\left(a_{y} b_{z}\right) \vec{\jmath} \vec{k}+\left(a_{z} b\right) \vec{k}+\left(a_{z} b_{x}\right) \vec{k} \vec{\imath} \\
+\left(a_{z} b_{y}\right) \vec{k} \vec{\jmath}+\left(a_{z} b_{z}\right) \vec{k} \vec{k} \\
=a b+\left(a b_{x}\right) \vec{\imath}+\left(a b_{y}\right) \vec{\jmath}+\left(a b_{z}\right) \vec{k}+\left(a_{x} b\right) \vec{\imath}-\left(a_{x} b_{x}\right)+\left(a_{x} b_{y}\right) \vec{k}-\left(a_{x} b_{z}\right) \vec{\jmath}+\left(a_{y} b\right) \vec{\jmath}-\left(a_{y} b_{x}\right) \vec{k} \\
-\left(a_{y} b_{y}\right)+\left(a_{y} b_{z}\right) \vec{\imath}+\left(a_{z} b\right) \vec{k}+\left(a_{z} b_{x}\right) \vec{\jmath}-\left(a_{z} b_{y}\right) \vec{\imath}-\left(a_{z} b_{z}\right) \\
=\left[a b-\left(a_{x} b_{x}\right)-\left(a_{y} b_{y}\right)-\left(a_{z} b_{z}\right)\right]+a\left[\left(b_{x}\right) \vec{\imath}+\left(b_{y}\right) \vec{\jmath}+\left(b_{z}\right) \vec{k}\right]+b\left[\left(a_{x}\right) \vec{\imath}+\left(a_{y}\right) \vec{\jmath}+\left(a_{z}\right) \vec{k}\right] \\
+\left[\left(a_{y} b_{z}\right)-\left(a_{z} b_{y}\right)\right] \vec{\imath}+\left[-\left(a_{x} b_{z}\right)+\left(a_{z} b_{x}\right)\right] \vec{\jmath}+\left[\left(a b_{z}\right)+\left(a_{x} b_{y}\right)-\left(a_{y} b_{x}\right)\right] \vec{k}
\end{gathered}
$$

Mas,

$$
\begin{gathered}
\vec{a} \times \vec{b}=\left|\begin{array}{ccc}
\vec{\imath} & \vec{\jmath} & \vec{k} \\
a_{x} & a_{y} & a_{z} \\
b_{x} & b_{y} & b_{z}
\end{array}\right|= \\
=\left(a_{y} b_{z}\right) \vec{\imath}+\left(a_{z} b_{x}\right) \vec{\jmath}+\left(a_{x} b_{y}\right) \vec{k}-\left(a_{y} b_{x}\right) \vec{k}-\left(a_{z} b_{y}\right) \vec{\imath}-\left(a_{x} b_{z}\right) \vec{\jmath} \\
=\left(a_{y} b_{z}-a_{z} b_{y}\right) \vec{\imath}+\left(a_{z} b_{x}-a_{x} b_{z}\right) \vec{\jmath}+\left(a_{x} b_{y}-a_{y} b_{x}\right) \vec{k}
\end{gathered}
$$

Portanto:

$$
\dot{a} \dot{b}=[a b-\vec{a} \vec{b}]+a \vec{b}+b \vec{a}+\vec{a} \times \vec{b}
$$

\section{Estrutura de Anéis}

Sejam $x, y, z \in \mathbb{R}$. Definimos que $(\mathbb{R},+)$ é um grupo se e somente se satisfazer as seguintes propriedades:

(i) Associatividade

$x+(y+z)=(x+y)+z$.

(ii) Existência do elemento neutro

$$
x+0=0+x=x .
$$

(iii) Existência do inverso

$x+(-x)=(-x)+x=0$.

Se $(\mathbb{R},+)$ satisfazer uma quarta operação, definida por

$$
x+y=y+x,
$$

dizemos que $(\mathbb{R},+)$ é um grupo comutativo.
Sejam $\quad x, y, z \in \mathbb{R}$. Definimos que $(\mathbb{R}, \cdot)$ é um grupo se e somente se satisfazer as seguintes operações:

(i) Associatividade

$$
(x y) z=x(y z) \text {. }
$$

(ii) Existência do elemento neutro

$$
x 1=1 x=x .
$$

(iii) Existência do inverso

$$
x \frac{1}{x}=x \frac{1}{x}=1 \text {, onde } x \neq 0
$$

(iv) Comutatividade

$$
x y=y x .
$$

(v) Distributividade

$$
x(y+z)=x y+x z .
$$


Definimos que $\mathbb{R}$ munido das operações de adição e multiplicação, satisfazendo a propriedade $(v)$, então $\left(\mathbb{R},+,{ }^{\prime}\right)$ é um anel. Se, além disso, for satisfeita também a propriedade (iv), dizemos que $(\mathbb{R},+,)^{\prime}$ é um anel comutativo.

\section{RESULTADOS}

Vamos verificar se o conjunto $\mathbb{H}$ munido das operações de adição e multiplicação é um anel através das propriedades anteriores.

Sejam $\dot{a}, \dot{b}$ e $\dot{c} \in \mathbb{H}$

- $(\mathbb{H},+)$ é um grupo se e somente se satisfazer as seguintes propriedades:

(i) Associatividade

$$
\dot{a}+(\dot{b}+\dot{c})=(\dot{a}+\dot{b})+\dot{c} .
$$

De acordo com equação 7.

(ii) Existência do elemento neutro

$$
\dot{a}+\dot{0}=\dot{0}+\dot{a}=\dot{a} .
$$

Onde $\dot{0}=(0, \overrightarrow{0})$.

(iii) Existência do inverso

$\dot{a}+(-\dot{a})=(-\dot{a})+\dot{a}=0$.

Onde $-\dot{a}=(-a,-\vec{a})$

Se $(\mathbb{H},+)$ satisfazer uma quarta operação, definida por

$$
\dot{a}+\dot{b}=\dot{b}+\dot{a},
$$

dizemos que $(\mathbb{H},+)$ é um grupo comutativo.

Portanto $(\mathbb{H},+)$ é um grupo comutativo.
- $(\mathbb{H}, \cdot)$ é um grupo se e somente se satisfazer as seguintes operações:

(i) Associatividade

$$
(\dot{a} \dot{b}) \dot{c}=\dot{a}(\dot{b} \dot{c}) .
$$

Conforme equação 8.

(ii) Existência do elemento neutro

$$
\dot{a} \dot{1}=\dot{1} \dot{a}=\dot{a} .
$$

Onde $\dot{1}=(1, \overrightarrow{0})$.

(iii) Existência do inverso

$$
\dot{a} \dot{a}^{-1}=\dot{a} \dot{a}^{-1}=1 .
$$

Onde $\dot{a}^{-1}=\frac{\dot{a}}{|\dot{a}|^{2}}$, para $\dot{a}^{-1} \neq 0$

(v) Distributividade

$$
\dot{a}(\dot{b}+\dot{c})=\dot{a} \dot{b}+\dot{a} \dot{c} .
$$

Contudo, (HH,') não é comutativo, conforme vimos em 3.

Desta forma, como $\mathbb{H}$ satisfaz as operações de $+e \cdot$, temos que $(\mathbb{H},+, \cdot)$ é um anel, não comutativo.

\section{DISCUSSÃO}

Os Quatérnions têm sido extensivamente utilizados em áreas como, por exemplo, Computação Gráfica, Geodésia, Visão Computacional, Fotogrametria e Robótica. Sua relevância se deve a sua associação com as rotações na forma dos ângulos de Euler no espaço e devido ao fato de apresentar algumas vantagens numéricas e ter um menor custo computacional. 


\section{CONCLUSÃO}

Os Quatérnions constituem uma teoria que, embora importante, é pouco difundida nos cursos de graduação em Matemática. Neste sentido, procuramos desenvolver o tema de forma acessível e com base em estruturas algébricas já conhecidas. Desta forma obtemos os Quatérnions como estrutura de Anel com divisão, pois não atende a propriedade comutativa. $\mathrm{O}$ anel dos Quatérnions pode ser visto como uma extensão do anel dos números complexos, com aplicações dentro e fora da Matemática.

\section{REFERÊNCIAS}

BOYER, C. B. História da matemática. São Paulo: Edgard Blücher, 1974.

BROUGHAM BRIDGE. 2004. Disponível em: <http://math.ucr.edu/home/baez/octonions/ node24.html>. Acesso em: 10 ago. 2015.

INGENIOUS IRELAND. Hamilton Quaternion walk - new podcast tour. 2011. Disponível em:

<http://ingeniousireland.ie/2011/11/hamilto n-quaternion-walk-new-podcast-tour/>. Acesso em: 10 ago. 2015. 